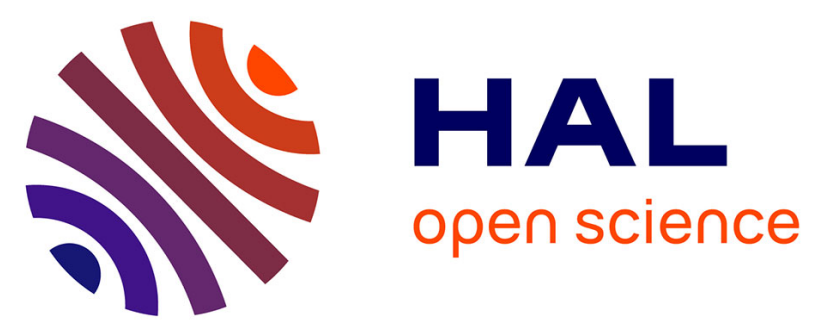

\title{
Practices in management of cancer treatment-related cardiovascular toxicity: A cardio-oncology survey
}

Ludovic Jovenaux, Jennifer Cautela, Noémie Resseguier, Michele Pibarot, Myriam Taouqi, Morgane Orabona, Johan Pinto, Michael Peyrol, Jeremie Barraud, Marc Laine, et al.

\section{To cite this version:}

Ludovic Jovenaux, Jennifer Cautela, Noémie Resseguier, Michele Pibarot, Myriam Taouqi, et al.. Practices in management of cancer treatment-related cardiovascular toxicity: A cardio-oncology survey. International Journal of Cardiology, 2017, 241, pp.387-392. 10.1016/j.ijcard.2017.02.154 . hal01798104

\section{HAL Id: hal-01798104 \\ https://hal-amu.archives-ouvertes.fr/hal-01798104}

Submitted on 23 May 2018

HAL is a multi-disciplinary open access archive for the deposit and dissemination of scientific research documents, whether they are published or not. The documents may come from teaching and research institutions in France or abroad, or from public or private research centers.
L'archive ouverte pluridisciplinaire HAL, est destinée au dépôt et à la diffusion de documents scientifiques de niveau recherche, publiés ou non, émanant des établissements d'enseignement et de recherche français ou étrangers, des laboratoires publics ou privés. 


\section{Practices in Management of Cancer Treatment-Related Cardiovascular}

\section{Toxicity: A Cardio-Oncology Survey}

Ludovic Jovenaux, MD, ${ }^{a, b, c, d}$ Jennifer Cautela, MD, ${ }^{a, b, c, d}$ Noemie Resseguier,MD, PhD, ${ }^{e}$

Michele Pibarot, MD, ${ }^{f}$ Myriam Taouqi, MD, ${ }^{f}$ Morgane Orabona, MD, ${ }^{a, b, c, d}$ Johan Pinto, MD, a,b,c,d Michael Peyrol, MD, ${ }^{a, b}$ Jeremie Barraud, MD, ${ }^{a, b}$ Marc Laine, MD, ${ }^{a, b}$ Laurent Bonello, MD, PhD, ${ }^{a, b}$ Franck Paganelli, MD, PhD ${ }^{a, b, c, d}$ Fabrice Barlesi, MD, PhD, , d, d, Franck Thuny, MD, $\mathrm{PhD},{ }^{\mathrm{a}, \mathrm{b}, \mathrm{c}, \mathrm{d}}$

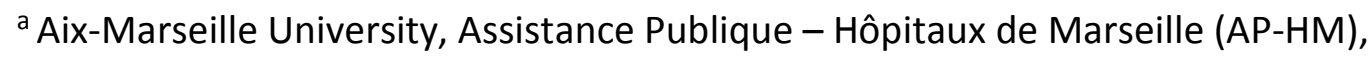
Mediterranean university Cardio-Oncology center (MEDI-CO center), Unit of Heart Failure and Valvular Heart Diseases, Department of Cardiology, Hôpital Nord, France ${ }^{\mathrm{b}}$ Mediterranean Association for Research and Studies in Cardiology (MARS Cardio), France ${ }^{c}$ Groupe Méditerranéen de Cardio-Oncologie (gMEDICO), France ${ }^{d}$ Aix-Marseille University, Assistance Publique - Hôpitaux de Marseille (AP-HM), Oncosaftey Network of the Early Phases Cancer Trials Center $($ CLIP²), France

e Aix-Marseille University, Department of Public Health, Research Unit EA 3279, France ${ }^{\text {f} A s s i s t a n c e ~ P u b l i q u e ~-~ H o ̂ p i t a u x ~ d e ~ M a r s e i l l e ~(A P-H M), ~ O N C O-P A C A-C O R S E ~ O n c o l o g y ~}$ Regional Network

g Aix-Marseille University, Multidisciplinary Oncology \& Therapeutic Innovations Department, Assistance Publique - Hôpitaux de Marseille (AP-HM), Hôpital Nord, France All the authors take responsibility for all aspects of the reliability and freedom from bias of the data presented and their discussed interpretation 


\section{Corresponding Author}

Prof. Franck Thuny

Mediterranean University Cardio-Oncology center (MEDI-CO center), Unit of Heart Failure and Valvular Heart Diseases, Hôpital NORD

Chemin des Bourrely, 13015, Marseille, France

Assistance Publique - Hôpitaux de Marseille (AP-HM), Aix-Marseille University

Tel: +33 (0) 491968683

Fax: +33 (0) 0491968979

e-mail: franck.thuny@gmail.com

Funding source: This research did not receive any specific grant from funding agencies in the public, commercial, or not-for-profit sectors.

Disclosures: Prof. Thuny reports receipt of consultancy fees from Philips, Astra-Zeneca, Daichy-Sankyo, General Electric, Novartis, Sanofi, Boston Scientific, and St Jude Medical outside the submitted work.

Key words: cardiotoxicity, cardio-oncology, cancer therapy, prevention, heart failure 


\section{ABSTRACT}

Background: Cardiovascular toxicity has become a challenging issue during cancer therapy. Nonetheless, there is a lack of consensual guidelines for their management. We aimed to determine the current practices of oncologists regarding cardiovascular toxicity related to anthracyclines, trastuzumab and angiogenic inhibitors and to gather their opinions on the development of cardio-oncology programs.

Methods: A cross-sectional declarative study was submitted to French oncologists in the form of an individual, structured questionnaire.

Results: A total of 303 oncologists responded to the survey. Ninety-nine percent of oncologists prescribed cardiotoxic therapies, including anthracyclines (83\%), trastuzumab (51\%) and other angiogenic inhibitors (64\%). The method adopted for managing cardiovascular toxicity was based on guidelines from expert oncology societies for only $35 \%$ of oncologists. None was aware of recommendations from expert cardiology societies. Prescription of pre-, peri- and post-therapy cardiovascular assessment was inconsistent and significantly less frequent for all classes of angiogenic inhibitors than for anthracyclines and trastuzumab $(P<0.0001)$. Relative to pre-therapy assessment, post-therapy assessment was prescribed significantly less often for all cancer therapies $(P<0.0001)$. Attitudes regarding the onset of left ventricular dysfunction were much more inconsistent when angiogenic inhibitors were involved. Additionally, the management of hypertension and QT prolongation was also inconsistent. Finally, $88 \%$ of oncologists supported projects of cardiooncology programs development.

Conclusions: Practices of oncologists are disparate in the field of cardiovascular toxicity. This finding underlines the complexity of managing many different situations and the need for 
distribution of formal guidelines from oncology and cardiology expert societies. The development of personalized cardio-oncology programs seems essential. 


\section{Abbreviations}

ASCO = American Society of Clinical Oncology

ASE/EACVI = American Society of Echocardiography/European Association of Cardio-Vascular Imaging

ESMO = European Society of Medical Oncology

VSP = Vascular endothelium growth factor pathway

$\mathrm{TTE}=$ transthoracic echocardiography 


\section{INTRODUCTION}

Cancer and cardiovascular diseases are the two leading causes of death in the United States and Europe, where they are responsible for almost $50 \%$ of overall mortality [1].

Cancer therapies are being rapidly developed and have improved the prognosis of many patients [2]. Nevertheless, the improvements in survival due to new cancer therapies bring a cost because these treatments can have deleterious effects on the cardiovascular system [3]. These include myocardial dysfunction, systemic hypertension, QT prolongation, arrhythmias, myocardial ischemia, pulmonary hypertension, thrombo-embolic events, accelerated atherosclerosis, pericardial and valvular heart diseases [4]. This toxicity can be observed very early or many years after the use of "old" classes of cytotoxic chemotherapy drugs or the most recent targeted therapies [3,5-7]. Furthermore, as a consequence of the aging population, cancer and cardiovascular diseases frequently co-exist, sometimes in an unknown manner $[8,9]$. Thus, cardiovascular toxicity management has become challenging because it could significantly influence global survival [10]. International guidelines on cardiovascular monitoring during and after cancer treatment lack consensus and are not based on substantial evidence, in particular for the novel targeted cancer therapies [11-16]. Additionally, the current practices regarding management of cardiovascular toxicity remain unknown.

Accordingly, we designed a national survey of French oncologists. The purpose of the study was to (1) analyze their professional practices in the field of prevention, screening, and treatment of cancer-therapy-related cardiovascular toxicity and (2) solicit their opinions on cardio-oncology programs that have recently emerged to manage cardiovascular diseases related to cancer treatments $[10,17-19]$. 


\section{METHODS}

\section{Study Design and Sample}

Between August 2015 and August 2016, we conducted a cross-sectional declarative survey in the form of an individual, structured questionnaire that was submitted to the 918 French medical oncologists working in teaching and non-teaching hospitals, cancer centres and private healthcare facilities in France (data from the French National Medical Council). The oncologists were invited to participate via regional oncology networks. The oncologists were first contacted by phone or e-mail to agree on a date for an interview, either in person or by phone, during which the answers to the survey questions were collected. They also had the opportunity to complete the questionnaire via an online version that was created for this purpose. For both paper- and web-based formats, we used an established method for questionnaire distribution to maximize response rates that involved a preliminary notice, an invitation letter with a questionnaire and up to 2 reminders, and another copy of the survey for non-responders. We merged all study data into a secure central database for analysis.

\section{Survey Instrument}

The questionnaire was composed of items written by four oncologists and four cardiologists specializing in cardio-oncology. The survey was arranged into three distinct sections, covering successively the following topics (Supplementary-file1):

Section (i): The profile of the oncologist and the organization of cardiovascular monitoring and management within their healthcare facility. This profile included specialization of the practitioner, fields of competence, place of work, professional experience and prescription of drugs with potential cardiovascular toxicity. It also discussed the physical presence within the structure of a cardiologist, a cardiology department, a cardiology care unit or a cardio- 
oncology unit, and the access within the structure of performing transthoracic echocardiography (TTE) studies. Our survey was focused on the most commonly used drugs with potential cardiovascular toxicity including anthracyclines, trastuzumab, vascular endothelium growth factor pathway (VSP) monoclonal antibodies and other angiogenic inhibitors.

Section (ii): The practice of the oncologist in the field of cardiovascular toxicity. Questions covered awareness of the existence of guidelines from expert societies and, in particular, the recommendations of the European Society of Medical Oncology (ESMO) [13] and the consensus of experts from the American Society of Echocardiography/European Association of Cardiovascular Imaging (ASE/EACVI) [14]. It also covered awareness of cardiovascular toxicity regarding the drugs prescribed, knowledge of screening methods and definitions of toxicity. Furthermore, it requested the cardiologist to estimate the number of patients who have presented with proven cardiovascular toxicity in association with a cancer therapy, the number of patients rejected from optimal cancer treatment because of a previous cardiovascular disease or risk factors and types of cardiovascular toxicity leading to interruption of cancer therapy. Finally, it covered modalities for pre-, peri- and post-therapy cardiovascular assessment, approaches to left ventricular dysfunction and prolongation of the QTc interval or hypertension.

Section (iii): Personal opinions regarding cardio-oncology programs; whether cardiooncology is of any value, openness to the opinion of a specialized cardiologist, the level of cardio-oncology expertise of the cardiologists with whom he or she works and whether there are obstacles to the creation or development of cardio-oncology programs. Information indicating that participation was voluntary and ensuring confidentiality was provided. Responding to the survey implied that the oncologists had given their consent to 
participate in the study.

\section{Statistical Analysis}

Categorical variables are described as counts and percentages with their $95 \%$ confidence intervals. The answers to the questions were expressed using a 5- or 6-category Likert scale. To facilitate presentation, certain responses were grouped together to form three categories. $\chi 2$ tests were used to compare the different groups. Statistical significance was defined as $P<0.05$. All tests were 2 -sided. Analyses were conducted using SPSS Statistics Software, version 20.0 (IBM Inc., New York, USA).

\section{RESULTS}

\section{Oncologists Profiles and Cardiovascular Management Organizations}

Of the 918 oncologists working in France, 303 responded to the survey. All regions of France were represented (Supplementary-file2). The profiles of oncologists and the organizations of cardiovascular management are summarized in Table 1. Three hundred and one oncologists (99\%) had prescribed potentially cardiotoxic cancer therapies, including anthracyclines (83\%), trastuzumab (51\%), VSP antibodies (63\%) and other angiogenic inhibitors (66\%). Eighty-three percent of the oncologists worked with cardiologists in the same healthcare facility. To assess left ventricular function, 283 oncologists (93\%) had used TTE, 156 (51\%) had used isotopic ventriculography, and 75 (25\%) had used cardiac MRI. 


\section{Global Practices in the Field of Cardiovascular Toxicity}

The method adopted by oncologists for managing the cardiovascular toxicity of cancer therapies was based essentially (52\%) on data obtained from the clinical trials conducted for each drug. Only 105 oncologists (35\%) declared that they used the guidelines of expert societies of oncology. None was aware of recommendations published by expert societies of cardiology in this field.

All the oncologists were aware that anthracyclines and trastuzumab could cause left ventricular dysfunction and heart failure. However, this toxicity was known to only $55 \%$ of oncologists for the VSP antibodies and $68 \%$ for the other angiogenic inhibitors. For these two types of angiogenic inhibitors, the main types of cardiovascular toxicity cited were hypertension (cited by $90 \%$ and $74 \%$ of the oncologists, respectively) and venous thromboembolic events ( $83 \%$ and $51 \%$ of the oncologists, respectively).

One hundred and fifteen oncologists (38\%) were unaware of the existence of early screening methods for left ventricular dysfunction. Using biomarkers such as troponin for this purpose or assessing global longitudinal strain in TTE were known methodologies to only $30 \%$ and $32 \%$ of oncologists, respectively. Seventy-five oncologists $(25 \%)$ were familiar with the use of cardiac MRI for this purpose. The upper limit of the QTc interval was unknown by 128 oncologists (42\%), and $180(60 \%)$ of them stated that they knew how to calculate it. Two hundred and seventy-nine oncologists (92\%) reported that they had been confronted at least once by proven cardiovascular toxicity associated with cancer therapy. Estimates of the annual incidence of the onset of this type of toxicity were $5 \%$ to $20 \%$ for $17 \%$ of the oncologists, and less than $5 \%$ for $75 \%$ of them. The most commonly cited forms of cardiovascular toxicity responsible for terminating a cancer therapy were left ventricular dysfunction with or without heart failure (84\%), venous thromboembolic events (56\%), 
coronary artery disease (35\%), hypertension (37\%) and QTc interval prolongation (18\%). Most of the respondents (93\%) had previously rejected a patient from a cancer therapy judged to be optimal because of a history of cardiovascular disease or risk factors.

\section{Cardiovascular Assessment}

The monitoring strategies according to the type of anti-cancer drug used are detailed in Table 2. A pre-therapy cardiovascular assessment (including cardiologic consultation and/or TTE) was prescribed systematically before use of anthracyclines and trastuzumab for $96 \%$ and $99 \%$ of oncologists, respectively. This pre-therapy assessment was significantly less frequent $(P<0.0001)$ for VSP antibodies $(43 \%)$ and other antiangiogenic drugs $(70 \%)$ (Figure 1A). A per-therapy cardiovascular assessment was prescribed systematically for trastuzumab by $95 \%$ of oncologists and significantly less often $(P<0.0001)$ for anthracyclines $(54 \%)$, VSP antibodies (28\%) and the other angiogenic inhibitors (42\%) (Figure 1B). When using anthracyclines, troponin serum level assessment was used by only $7 \%$ of oncologists. The post-therapy cardiovascular assessment (at the end of the treatment and thereafter) was prescribed systematically more often for anthracyclines (49\%) and trastuzumab (59\%) than for VSP antibodies (14\%) and the other angiogenic inhibitors $(28 \%)(P<.0001)$ (Figure 1C). Relative to pre-therapy cardiovascular assessment, post-therapy assessment was prescribed significantly less often for all therapies, including anthracyclines ( $96 \%$ versus 49\%, $P<0.0001)$, trastuzumab (99\% versus $59 \%, P<.0001)$, VSP antibodies ( $43 \%$ versus $14 \%$, $P<0.0001)$, and the other angiogenic inhibitors (70\% versus $28 \%, P<0.0001)$. 


\section{Management of Left Ventricular Dysfunction}

The approach to the onset of left ventricular dysfunction (a decrease in LVEF) in relation to the type of cancer therapy used is described in Figure 2. For anthracyclines, management was inconsistent for asymptomatic patients with an LVEF between 40 and $50 \%$ and relatively consistent in all other cases (Figure 2A).

For trastuzumab, the management was inconsistent for all cases unless for asymptomatic patients with an LVEF between $40 \%$ and 50\% (Figure 2B).

For VSP antibodies and other angiogenic inhibitors, management was inconsistent in all cases of left ventricular dysfunction (Figure 2C and Figure 2D).

\section{Management of Hypertension and QT Prolongation}

In case of hypertension under all angiogenic inhibitors, excluding cases of hypertensive emergency, $82 \%$ of oncologists performed systematic screening for proteinuria, and $44 \%$ chose to introduce an antihypertensive treatment themselves. Additionally, only $27 \%$ of oncologists maintained the cancer therapy, while $40 \%$ opted for a temporary interruption while waiting for a cardiology consultation. One third of the oncologists stated that they were unfamiliar with the approach to this situation.

When confronted with a >500 msec QTc interval prolongation or $>60$ msec progression, $45 \%$ of oncologists declared that they did not know how to manage the situation. In all the cases, the opinion of a cardiologist was requested.

\section{Cardio-Oncology Programs}

Sixty-four percent of oncologists believed that with regard to the cardiovascular toxicity of cancer therapies, the level of knowledge of the cardiologists with whom they worked could 
be improved, and $26 \%$ believed that this knowledge was insufficient. Most of the oncologists (88\%) supported the idea that the presence within their healthcare facilities of a cardiooncology unit or using a cardiologist specialized in this field could improve their management of the cardiovascular adverse events. The primary obstacle to the creation or extension of cardio-oncology programs within their establishments was of a financial nature for $61 \%$ of oncologists. Limitations in terms of insufficient infrastructure were mentioned in $22 \%$ of cases, while $27 \%$ of the oncologists feared that the interest provoked by the creation of this program would be insufficient, particularly for the cardiologists with whom they work. Finally, 22\% of the oncologists believed that no real obstacle existed.

\section{DISCUSSION}

This study is the first to analyze the current practices of oncologists in the challenging field of cancer-treatment-related cardiovascular toxicity. In the context of lack of consensual guidelines [11-16], this survey showed that the assessment and management of the cardiovascular status of cancer patients is often inconsistent between oncologists before, during and after cancer therapy administration. While the great majority of respondents prescribed potentially cardiotoxic drugs, their attitude was particularly disparate toward the use of angiogenic inhibitors in comparison with "older" chemotherapy drugs such as anthracyclines. Finally, their opinion was favorable regarding the development of cardiooncology programs.

Regarding the cardiac monitoring in relation to prescribed treatments, we observed that the management was relatively consistent when anthracyclines or trastuzumab were used, whereas it was notably inconsistent with the use of angiogenic inhibitors. This result can be 
explained by a better overall knowledge of the incidence and the mechanisms of adverse cardiac side effects that may occur during the use of anthracyclines or trastuzumab. In fact, the results of numerous clinical trials have led to the standardization of monitoring during use of these treatments $[2,13,20]$. Instead, the different responses collected for monitoring under angiogenic inhibitors can be explained by much more disparate data regarding the incidence of life-threatening cardiac adverse effects such as heart failure [7,21]. Recently, the guidelines from the American Society of Clinical Oncology (ASCO) estimated that there is insufficient evidence to recommend a specific cardiac monitoring for patients treated by these drugs [16] whereas the European Society of Cardiology considers reasonable to perform TTE every 6 months until stability in LVEF values is achieved [15]. Although global longitudinal strain measurement and biomarkers such as serum troponin have shown promise as potential early biomarkers of cardiac dysfunction [22,23], the respondents seemed unfamiliar with these tools. Their advantages have been reported in many recent recommendations but without strong level of evidence [22-25]. Regarding the approach to left ventricular dysfunction, we found differences between anthracyclines and trastuzumab on the one hand and angiogenic inhibitors on the other hand. The disparity of the approaches when using angiogenic inhibitors can be explained by the same arguments as those cited above for patient monitoring. Nevertheless, different responses to the approach to left ventricular dysfunction were also observed when using anthracyclines and trastuzumab, particularly in difficult cases where the patient is asymptomatic with LVEF between $40 \%$ and $50 \%$. In this case, the ESMO recommends holding anthracyclines but continuing trastuzumab [13]. In the survey, only $38 \%$ of oncologists using anthracyclines and $21 \%$ using trastuzumab adopted this recommended approach. However, guidelines oversimplify what is always a complex decision. Decisions regarding continuing, 
holding, or discontinuing cancer therapy should be made based on a risk-benefit balance weighing how significant the cancer treatment is to the patient (benefit) versus the totality of the risks. For example, one would be willing to tolerate mild cardiac toxicity in a patient with cancer therapy effective for a metastatic tumor, whereas one would be likely to tolerate much less toxicity for an adjuvant oncology treatment with relatively modest benefit. Thus, the ASCO guidelines recently stated that no recommendations could be made regarding continuation or discontinuation of cancer therapy in individuals with evidence of cardiac dysfunction. The disparate responses in difficult cases illustrate the need for close collaboration between oncologists and cardiologists to ensure the most appropriate treatment decisions for each patient.

Systemic hypertension represents the most common cardiovascular complication associated with angiogenic inhibitors [26]. This outcome can be linked to glomerular disease and renal thrombotic microangiopathy, thus justifying proteinuria screening and follow-up of the glomerular filtration rate. In our survey, this complication was well known to most of the respondents. Thus, the systematic search for proteinuria was routine in the vast majority of cases. However, regarding the continuation or cessation of angiogenic inhibitors, most oncologists (73\%) did not have a prepared response or interrupted treatment pending cardiology consultation. Indeed, as reported by The Cardiovascular Toxicities Panel of the National Cancer Institute, there is no single algorithm to follow in managing hypertension induced by angiogenic inhibitors and treatment needs to be individualized for each patient. Again, this is an expert opinion based on consensus rather than on clinical outcome data [27].

Most of the respondents supported the idea that a cardio-oncology unit or the presence of a specialized cardio-oncologist in their healthcare facilities could improve their management 
of adverse cardiovascular events related to cancer therapies. Similar opinion was recently reported among cardiologists [28]. Actually, in response to the challenging management of cardiovascular toxicity, the development of the cardio-oncology sub-specialty might be considered a helpful solution. Through a multidisciplinary approach, this specialty aims to provide optimal care for patients with cardiovascular diseases or relevant risk factors from the time of cancer diagnosis and throughout their lives, even after the completion of treatment. The oncologists' opinions toward the development of cardio-oncology programs seemed to be favorable, particularly because most of them believed that access to cardiologists was not always possible and that their level of knowledge in cardiovascular adverse events could be improved. Over the past few years, medical centers in the United States, Canada, and Europe have developed cardio-oncology programs $[3,19,29]$. This development has allowed for better coordination in the management of patients because of easier access to comprehensive cardiovascular assessment by specialized cardiologists and better assessment of the therapeutic benefit-to-risk ratio of cancer treatments by closer communication between cardiologists and oncologists. This partnership has been supported by action from different professional scientific societies $[15,30]$.

The results of our survey should be regarded in light of potential study limitations. We could not compare the profiles of respondents versus those of non-respondents. Moreover, this was a declarative survey, and the nature of the topics explored may have led to response bias, including a difference between declared practice and actual practice, and underreporting of negative opinions on the development of cardio-oncology programs. To minimize this potential bias, we provided full assurances of confidentiality. Furthermore, proposed answers sometimes were not appropriated in all clinical situations encountered in practice. For example, the answers would have been different according to the oncological 
or the cardiologic profiles of patients. Finally, this survey was performed in a single European country and may not reflect the practices elsewhere.

This study presents an overview of oncologists' practices regarding the challenging field of cancer treatment-related cardiovascular toxicity. It shows major disparities in the approaches underlining the complexity of management of many different situations and the need for distribution of consensual guidelines involving both oncology and cardiology expert societies. Finally, the management of cancer patients might be improved by a multidisciplinary approach and equal access to innovative and personalized cardio-oncology programs. 


\section{REFERENCES}

1 Kochanek KD, M. S., Xu J, Arias E: Mortality in the United States, 2013. NCHS Data Brief.2014; 178:1-8

2 Goldhirsch, A., Gelber, R. D., Piccart-Gebhart, M. J., de Azambuja, E., Procter, M., Suter, T. M. et al.: 2 years versus 1 year of adjuvant trastuzumab for HER2-positive breast cancer (HERA): an open-label, randomised controlled trial. Lancet.2013; $382: 1021-1028$

3 Cautela, J., Lalevee, N., Ammar, C., Ederhy, S., Peyrol, M., Debourdeau, P. et al.: Management and research in cancer treatment-related cardiovascular toxicity: Challenges and perspectives. Int J Cardiol.2016; 224:366-375

4 Curigliano, G., Cardinale, D., Dent, S., Criscitiello, C., Aseyev, O., Lenihan, D. et al.: Cardiotoxicity of anticancer treatments: Epidemiology, detection, and management. CA Cancer J Clin.2016;

5 Moslehi, J. J.: Cardiovascular Toxic Effects of Targeted Cancer Therapies. N Engl J Med.2016; 375:1457-1467

6 Cardinale, D., Colombo, A., Bacchiani, G., Tedeschi, I., Meroni, C. A., Veglia, F. et al.: Early detection of anthracycline cardiotoxicity and improvement with heart failure therapy. Circulation.2015; 131:1981-1988

7 Ewer, M. S., Suter, T. M., Lenihan, D. J., Niculescu, L., Breazna, A., Demetri, G. D. et al.: Cardiovascular events among 1090 cancer patients treated with sunitinib, interferon, or placebo: a comprehensive adjudicated database analysis demonstrating clinically meaningful reversibility of cardiac events. Eur J Cancer.2014; $50: 2162-2170$ 
8 Koene, R. J., Prizment, A. E., Blaes, A. \& Konety, S. H.: Shared Risk Factors in Cardiovascular Disease and Cancer. Circulation.2016; 133:1104-1114

9 Hasin, T., Gerber, Y., Weston, S. A., Jiang, R., Killian, J. M., Manemann, S. M. et al.: Heart Failure After Myocardial Infarction Is Associated With Increased Risk of Cancer. J Am Coll Cardiol.2016; 68:265-271

10 Yeh, E. T. \& Chang, H. M.: Oncocardiology-Past, Present, and Future: A Review. JAMA Cardiol.2016;

11 Armenian, S. H., Hudson, M. M., Mulder, R. L., Chen, M. H., Constine, L. S., Dwyer, M. et al.: Recommendations for cardiomyopathy surveillance for survivors of childhood cancer: a report from the International Late Effects of Childhood Cancer Guideline Harmonization Group. Lancet Oncol.2015; 16:e123-136

12 Lancellotti, P., Nkomo, V. T., Badano, L. P., Bergler-Klein, J., Bogaert, J., Davin, L. et al.: Expert consensus for multi-modality imaging evaluation of cardiovascular complications of radiotherapy in adults: a report from the European Association of Cardiovascular Imaging and the American Society of Echocardiography. Eur Heart J Cardiovasc Imaging.2013; 14:721-740

13 Curigliano, G., Cardinale, D., Suter, T., Plataniotis, G., de Azambuja, E., Sandri, M. T. et al.: Cardiovascular toxicity induced by chemotherapy, targeted agents and radiotherapy: ESMO Clinical Practice Guidelines. Ann Oncol.2012; 23 Suppl 7:vii155166

14 Plana, J. C., Galderisi, M., Barac, A., Ewer, M. S., Ky, B., Scherrer-Crosbie, M. et al.: Expert consensus for multimodality imaging evaluation of adult patients during and after cancer therapy: a report from the American Society of Echocardiography and 
the European Association of Cardiovascular Imaging. J Am Soc Echocardiogr.2014; 27:911-939

15 Zamorano, J. L., Lancellotti, P., Rodriguez Munoz, D., Aboyans, V., Asteggiano, R., Galderisi, M. et al.: 2016 ESC Position Paper on cancer treatments and cardiovascular toxicity developed under the auspices of the ESC Committee for Practice Guidelines: The Task Force for cancer treatments and cardiovascular toxicity of the European Society of Cardiology (ESC). Eur Heart J.2016; 37:2768-2801

16 Armenian, S. H., Lacchetti, C., Barac, A., Carver, J., Constine, L. S., Denduluri, N. et al.: Prevention and Monitoring of Cardiac Dysfunction in Survivors of Adult Cancers: American Society of Clinical Oncology Clinical Practice Guideline. J Clin Oncol.2016:JCO2016705400

17 Moslehi, J. \& Cheng, S.: Cardio-oncology: it takes two to translate. Sci Transl Med.2013; 5:187fs120

18 Abdel-Qadir, H., Amir, E. \& Thavendiranathan, P.: Prevention, Detection, and Management of Chemotherapy-Related Cardiac Dysfunction. Can J Cardiol.2016; 32:891-899

19 Parent, S., Pituskin, E. \& Paterson, D. I.: The Cardio-oncology Program: A Multidisciplinary Approach to the Care of Cancer Patients With Cardiovascular Disease. Can J Cardiol.2016; 32:847-851

20 Tan-Chiu, E., Yothers, G., Romond, E., Geyer, C. E., Jr., Ewer, M., Keefe, D. et al.: Assessment of cardiac dysfunction in a randomized trial comparing doxorubicin and cyclophosphamide followed by paclitaxel, with or without trastuzumab as adjuvant therapy in node-positive, human epidermal growth factor receptor 2-overexpressing breast cancer: NSABP B-31. J Clin Oncol.2005; 23:7811-7819 
21 Chu, T. F., Rupnick, M. A., Kerkela, R., Dallabrida, S. M., Zurakowski, D., Nguyen, L. et al.: Cardiotoxicity associated with tyrosine kinase inhibitor sunitinib. Lancet.2007; $370: 2011-2019$

22 Cardinale, D., Sandri, M. T., Colombo, A., Colombo, N., Boeri, M., Lamantia, G. et al.: Prognostic value of troponin I in cardiac risk stratification of cancer patients undergoing high-dose chemotherapy. Circulation.2004; 109:2749-2754

23 Sawaya, H., Sebag, I. A., Plana, J. C., Januzzi, J. L., Ky, B., Tan, T. C. et al.: Assessment of echocardiography and biomarkers for the extended prediction of cardiotoxicity in patients treated with anthracyclines, taxanes, and trastuzumab. Circ Cardiovasc Imaging.2012; 5:596-603

24 Cardinale, D., Colombo, A., Sandri, M. T., Lamantia, G., Colombo, N., Civelli, M. et al.: Prevention of high-dose chemotherapy-induced cardiotoxicity in high-risk patients by angiotensin-converting enzyme inhibition. Circulation.2006; 114:2474-2481

25 Kang, Y., Xu, X., Cheng, L., Li, L., Sun, M., Chen, H. et al.: Two-dimensional speckle tracking echocardiography combined with high-sensitive cardiac troponin $\mathrm{T}$ in early detection and prediction of cardiotoxicity during epirubicine-based chemotherapy. Eur J Heart Fail.2014; 16:300-308

26 Li, W., Croce, K., Steensma, D. P., McDermott, D. F., Ben-Yehuda, O. \& Moslehi, J.: Vascular and Metabolic Implications of Novel Targeted Cancer Therapies: Focus on Kinase Inhibitors. J Am Coll Cardiol.2015; 66:1160-1178

27 Maitland, M. L., Bakris, G. L., Black, H. R., Chen, H. X., Durand, J. B., Elliott, W. J. et al.: Initial assessment, surveillance, and management of blood pressure in patients receiving vascular endothelial growth factor signaling pathway inhibitors. I Natl Cancer Inst.2010; 102:596-604 
28 Barac, A., Murtagh, G., Carver, J. R., Chen, M. H., Freeman, A. M., Herrmann, J. et al.: Cardiovascular Health of Patients With Cancer and Cancer Survivors: A Roadmap to the Next Level. J Am Coll Cardiol.2015; 65:2739-2746

29 Okwuosa, T. M., Akhter, N., Williams, K. A. \& DeCara, J. M.: Building a cardiooncology program in a small- to medium-sized, nonprimary cancer center, academic hospital in the USA: challenges and pitfalls. Future Cardiol.2015; 11:413-420

30 Virani, S. A., Dent, S., Brezden-Masley, C., Clarke, B., Davis, M. K., Jassal, D. S. et al.: Canadian Cardiovascular Society Guidelines for Evaluation and Management of Cardiovascular Complications of Cancer Therapy. Can J Cardiol.2016; 32:831-841 


\section{TABLES}

Table 1. Profile of the Doctors and Organization of Cardiology Management ( $N=303)$

Characteristics of the doctor

No. (\%) of oncologists (\%)

Place of work

Public university teaching hospitals

$146(48)$

Public non-teaching hospitals

$37(12)$

Cancer centers

$95(32)$

Private clinics

$22(7)$

Private Healthcare establishments of Collective Interest

Fields of competence

Breast health and gynecology

$122(40)$

Hematology

$88(29)$

Gastroenterology

$76(25)$

Pneumology

$68(22)$

Urology

$67(22)$

Ear-Nose-Throat

$45(15)$

Dermatology

37 (12)

Neurology

Sarcomas

Specializations

Medical oncology

$121(40)$

Onco-hematology

7 (24)

Radiotherapy

$30(10)$

Organ oncology

Other specializations

Length of practice

$<10$ years

$119(40)$

10 to 20 years

93 (32)

$>20$ years

$81(28)$

Anti-cancer therapies prescribed

Anthracyclines

$250(83)$

Trastuzumab

$154(51)$

VSP antibodies

$188(63)$

Other angiogenic inhibitors

$198(66)$

Other targeted therapies

$139(46)$

Hormone therapy

$117(39)$

Immunotherapy/check point inhibitors

$114(38)$

Radiotherapy

$102(34)$

Collaboration with cardiology

Cardiologists working in the same healthcare facility

$250(83)$

Cardiology department in the same healthcare facility

$183(60)$

External cardiologists coming in to work in the healthcare facility

$67(22)$

Cardiologists with an ultrasound machine at their disposition at the

$227(75)$ consultation 
The totals may reach a figure of less than 303 because of the lack of response to certain items. VSP, vascular endothelium growth factor signal pathway. 
Table 2. Cardiology Monitoring Strategy for Cancer treatments as Evaluated by the Question: "Do you prescribe cardiologic assessment when using any of these compounds?"
Never
Patients at risk
Always

Pre-therapy monitoring

No. $(\%)$ of oncologists $[95 \% \mathrm{Cl}] \quad$ No. $(\%)$ of oncologists $[95 \% \mathrm{Cl}] \quad$ No. $(\%)$ of oncologists $[95 \% \mathrm{Cl}]$

Anthracyclines

Trastuzumab

VSP antibodies

1 (1) [0-2.2]

$$
0 \text { (0) }
$$

15 (9) [4.7-13.3]

1 (1) [0-2.4]

Monitoring during therapy

Anthracyclines

Trastuzumab

VSP antibodies

Other angiogenic inhibitors

\section{Post-therapy monitoring}

Anthracyclines

Trastuzumab

VSP antibodies

Other anti-angiogenics
9 (4) [1.6-6.4]

2 (1) [0-2.6]

26 (15) [9.6-20.4]

5 (5) [1.9-8.1]

37 (15) [10.6-19.4]

24 (16) [10.2-21.8]

50 (29) [22.2-35.8]

$$
\begin{gathered}
8(3)[0.9-5.1] \\
1(1)[0-2.6] \\
83(48)[40.5-55.5] \\
30(29)[22.5-35.5] \\
105(42)[35.9-48.1] \\
6(4)[0.9-7.1] \\
98(57)[49.6-64.4] \\
55(53)[45.9-60.1]
\end{gathered}
$$

90 (36) [30-42]

38 (25) [18.2-31.8]

98 (57) [49.6-64.4]

$60(58)$ [50.9-65.1]
241 (96) [93.6-98.4]

152 (99) [97.4-100]

74 (43) [35.6-50.4]

73 (70) [63.4-76.6]

136 (54) [47.8-60.2]

145 (95) [91.6-98.4]

48 (28) [21.3-34.7]

$44(42)$ [34.9-49.1]

123 (49) [42.8-55.2]

91 (59) [51.3-66.7]

24 (14) [8.8-19.2]

29 (28) [21.6-34.4]

The totals may reach a figure of less than 303 because of the lack of response to certain items. $\mathrm{Cl} 95 \%$, $95 \%$ confidence interval; VSP, vascular endothelium growth factor signal pathway. 


\section{FIGURES LEGENDS}

Figure 1. Pre- (A), Per- (B), and Post-Therapy (C) Cardiovascular Assessment According to the Prescribed Cancer Treatments

Results are expressed as percentage and 95\% confidence interval. VSP, vascular endothelium growth factor signal pathway.

Figure 2. Approach to the Onset of Left Ventricular Dysfunction in Relation to its Severity and the Type of Cancer Therapy Used

Results are expressed as percentage and 95\% confidence interval. HF, heart failure; LVEF, left ventricular ejection fraction; VSP, vascular endothelium growth factor signal pathway. 